\title{
AUTOMATIC CONTINUITY OF HOMOMORPHISMS IN TOPOLOGICAL ALGEBRAS
}

\author{
S. J. BHATT
}

(Communicated by Palle E. T. Jorgensen)

\begin{abstract}
A homomorphism from a locally convex $Q$-algebra to a uniform topological algebra is continuous. A one-to-one homomorphism from a regular complete spectrally bounded uniform topological algebra onto a dense subalgebra of a semisimple locally $m$-convex $Q$-algebra is open. Examples are discussed to show that none of the assumptions in these results can be omitted.
\end{abstract}

\section{PReliminaries AND NOtATION}

A uniform seminorm on a linear associative algebra $A$ (over complex scalars) is a seminorm $p$ satisfying (i) $p(x y) \leq p(x) p(y)$ for all $x, y$, and (ii) $p\left(x^{2}\right)=$ $p(x)^{2}$ for all $x$. A (locally convex) topological algebra [8] is an algebra $A$ with a Hausdorff topology $t$ on it so that $(A, t)$ is a (locally convex) topological vector space in which the multiplication is separately continuous. It is a $Q$ algebra [9, Appendix E] if the set of all quasi-regular elements is an open set. A locally $m$-convex algebra (lmc algebra) [9] is a locally convex topological algebra whose topology is determined by a separating family $P=\left(p_{\alpha}\right)$ of seminorms each satisfying (i). For each $\alpha$, let $N_{\alpha}=\left\{x \in A \mid p_{\alpha}(x)=0\right\}$ and $A_{\alpha}$ be the Banach algebra obtained by completing $A / N_{\alpha}$ in the norm $\left\|x_{\alpha}\right\|_{\alpha}=p_{\alpha}(x)$, $x_{\alpha}=x+N_{\alpha}$. If $A$ is complete, then $A$ is an inverse limit of Banach algebras $A=\lim A_{\alpha}[9$, Theorem 5.1]. A uniform topological algebra (uT-algebra) [3] is an $\operatorname{Imc}$ algebra $A$ in which each $p_{\alpha}$ additionally satisfies (ii) so each $A_{\alpha}$ is a uniform Banach algebra. A uniform Banach algebra ( $u B$-algebra) is a Banach algebra $(A,\|\cdot\|)$ such that $\left\|x^{2}\right\|=\|x\|^{2}$ for all $x$. By [4, Theorem 3.10, p. 32], a uB-algebra is commutative; via Gelfand theory, it is a closed point separating subalgebra of the supnorm Banach algebra $C(X)$ of all continuous complexvalued functions on a compact Hausdorff space $X$. Thus, a uT-algebra $A$ is commutative, and if complete, $A$ is an inverse limit of uB-algebras. An algebra $A$ is spectrally bounded $(s b)$ if for each $x \in A$ its $\operatorname{spectrum} \operatorname{sp}_{A}(x)$ in $A$ is a bounded subset of the complex plane. Throughout, $r(x) \quad\left(=r_{A}(x)\right)$ denotes the spectral radius of $x$ in $A$. The bounded part of a uT-algebra $A$ is the

Received by the editors January 21, 1992.

1991 Mathematics Subject Classification. Primary 46H40.

Key words and phrases. Uniform seminorm, locally convex $Q$-algebras, uniform algebras, regular algebras. 
subalgebra $b(A)=\left\{x \in A \mid \sup _{\alpha} p_{\alpha}(x)<\infty\right\}$. If $A$ is complete, then $b(A)$ is a uB-algebra with norm $\|x\|_{\infty}=\sup _{\alpha} p_{\alpha}(x)$ continuously embedded in $A$ [3]. For a commutative lmc algebra $A$, let $\sigma(A)$ denote the Gelfand space of $A$ consisting of all nonzero continuous multiplicative linear functionals with the relative weak* topology. $A$ is semisimple if, for any $x \in A, f(x)=0$ for all $f \in \sigma(A)$ implies that $x=0$. As in Banach algebras [8, Chapter 7], $A$ is regular if given a closed subset $F \subset \sigma(A)$ and $f \in \sigma(A), f \notin F$, there exists an $x \in A$ such that $\left.\hat{x}\right|_{F}=0$ and $\hat{x}(f) \neq 0, \hat{x}: \sigma(A) \rightarrow \mathbb{C}, \hat{x}(g)=g(x)$, is the Gelfand transform of $x$.

\section{MAIN RESUlts}

Theorem 2.1. Let $A$ be an sb algebra, $B$ a uT-algebra, and $\phi: A \rightarrow B$ a homomorphism. Then $\phi(A) \subset b(B)$, and, for each continuous uniform seminorm $q$ on $B, q(\phi(x)) \leq r(x)$ for all $x$ in $A$. In particular, if $A$ is a locally convex $Q$-algebra, then $\phi$ is continuous.

Theorem 2.2. Let $A$ be an $s b$, regular, complete, uT-algebra and $B$ be an Imc algebra. Let $\phi: A \rightarrow B$ be a one-to-one homomorphism such that $\overline{\operatorname{Im}(\phi)}$ is a semisimple $Q$-algebra. Then $\left.\phi^{-1}\right|_{\operatorname{Im}(\phi)}$ is continuous.

Note that, in the theorems, in the absence of metrizability and completeness, automatic continuity is guaranteed by a ring theoretic condition of topological nature. In $\S 3$ we discuss several examples exhibiting that various assumptions in the theorems cannot be omitted.

Proof of Theorem 2.1. We can assume $B$ to be complete since it is easy to verify that the completion of a uT-algebra is a uT-algebra. Also, the topology of $B$ is determined by the collection $S(B)$ of all continuous uniform seminorms on $B$; thus, $B=\lim _{q \in S(B)} B_{q}$, where $B_{q}$ is the uB-algebra obtained by completing $\left.B / N_{q} \quad\left(N_{q}=\overleftarrow{\{x} \in B \mid q(x)=0\right\}\right)$ in the norm $\left\|y_{q}\right\|_{q}=q(y), y_{q}=y+N_{q}$. Then $b(B)=\left\{y \in B \mid \operatorname{sp}_{B}(y)\right.$ is bounded $\}$. Indeed, $B$ being complete and Imc, [9, Corollary 5.3] implies that, for each $y \in B, \operatorname{sp}_{B}(y)=\bigcup\left\{\operatorname{sp}_{B_{q}}\left(y_{q}\right) \mid q \in\right.$ $S(B)\}$ and $r_{B}(y)=\sup _{q \in S(B)} \lim \sup _{n \rightarrow \infty} q\left(y^{n}\right)^{1 / n}=\sup _{q \in S(B)} q(y)$ in view of $q\left(y^{2}\right)=q(y)^{2}$. Now let $x \in A, q \in S(B)$. Since $\operatorname{sp}_{A}(x) \supset \operatorname{sp}_{B}(\phi(x))$, it follows that $q(\phi(x))=\left\|(\phi(x))_{q}\right\|_{q}=r_{B_{q}}\left(\phi(x)_{q}\right) \leq r_{B}(\phi(x)) \leq r_{A}(x)<\infty$; moreover, $\phi(A) \subset b(B)$. Further, assume $A$ to be a $Q$-algebra (so that it is sb by [9, Lemma E3]) which is also locally convex. By [9, Proposition 13.5] $s(A)=\{x \in A \mid r(x)<1\}$ is a neighbourhood of 0 ; hence there exists a convex, balanced, open set $U \subset A$ such that $0 \in U \subset s(A)$. Let $p=p_{U}$ be the Minkowski functional of $U$ in $A$; it is a continuous seminorm. As in the proof of [10, Theorem 1.36], $U=\{x \in A \mid p(x)<1\}$. For $x \in A, \delta>0$, $p(y)<1$, where $y=x /(p(x)+\delta)$. Thus $y \in s(A)$; hence, $r_{A}(x)<p(x)+\delta$. This gives $q(\phi(x)) \leq r_{A}(x)=\sup _{q \in S(B)} q(\phi(x)) \leq p(x)$, showing that $\phi$ is continuous.

Remark. Since $q \in S(B)$ is arbitrary, it follows that

$$
\|\phi(x)\|_{\infty}=\sup _{q \in S(B)} q(\phi(x)) \leq p(x) \quad(x \in A),
$$

giving the stronger assertion that $\phi: A \rightarrow\left(b(B),\|\cdot\|_{\infty}\right)$ is continuous. 
Proof of Theorem 2.2. It follows by the description of the bounded part of a uT-algebra in the first proof that $A=b(A)$ as sets. We can assume, without loss of generality, that $A$ possesses an identity 1 ; thus, $\sigma(A)$ is a compact Hausdorff space. As $\overline{\operatorname{Im}(\sigma)}$ is a (commutative) lmc $Q$-algebra, the inversion is continuous; hence, [7, Proposition 1.6, p. 168] implies that $\sigma(\overline{\operatorname{Im}(\phi)})=\sigma(\operatorname{Im}(\sigma))$ is also a compact Hausdorff space. Let $C=\overline{\operatorname{Im}(\phi)}$. Now the adjoint map $\phi^{*}: \sigma(C) \rightarrow$ $\sigma(A), \phi^{*}(f)=f \circ \phi$ is continuous; hence, $F=\phi^{*}(\sigma(C))$ is closed in $\sigma(A)$. In fact, $\phi^{*}$ is surjective because if not, regularity of $A$ implies that there exists an $x \in A, x \neq 0$, such that $\left.\hat{x}\right|_{F}=0$; thus, $\phi^{*}(f)(x)=f(\phi(x))=0$ for all $f \in \sigma(C)$. Since $C$ is semisimple, $\phi(x)=0$, contradicting that $\phi$ is one-toone. Thus $F=\sigma(A)$. Then by [9, Corollary 5.6], for any $x \in A, \operatorname{sp}_{A}(x)=$ $\{f(x) \mid f \in \sigma(A)\}=\{f(\phi(x)) \mid f \in \sigma(C)\}=\operatorname{sp}_{C}(\phi(x))$. Now $C$ being an lmc $Q$-algebra, there exists a continuous seminorm $q$ on $C$ such that $r_{C}(\phi(x)) \leq$ $q(\phi(x))(x \in A)$ [9, Proposition 13.5]. Then for any $p \in S(A), x \in A$, $p(x)=\left\|x_{p}\right\|_{p}=r_{A_{p}}(x) \leq r_{A}(x)=r_{C}(\phi(x)) \leq q(\phi(x))$, showing that $\left.\phi^{-1}\right|_{(\operatorname{Im}(\phi))}$ is continuous.

Corollary 2.3. Let $A$ be a unital locally convex $Q$-algebra. Then every uniform seminorm $\rho$ on $A$ is continuous. Further, if the inversion in $A$ is continuous, then $p(x) \leq r(x)$ for all $x$.

Proof. The quotient map $\phi: A \rightarrow\left(A_{p},\|\cdot\|_{p}\right), \phi(x)=x_{p}$, is continuous by Theorem 2.1, and there exists a continuous seminorm $q$ on $A$ such that, for all $x \in A, p(x)=\|\phi(x)\|_{p} \leq q(x)$. In fact, $p(x)=\lim _{n} p\left(x^{2^{n}}\right)^{1 / 2^{n}} \leq$ sup lim sup $\sin _{n \rightarrow \infty} q\left(x^{2^{n}}\right)^{1 / 2^{n}}=\beta(x)$ [1, Theorem 3.12], where $\beta(x)$ is the radius of boundedness in the sense of [1], but $\beta(x) \leq r(x)$ again by [1, Theorem 3.12]. (The assumption that the inversion map on $A$ is continuous is required [1, Theorem 4.1] for the equality of the spectral radii for the usual spectrum considered here and the spectrum considered in [1].)

Corollary 2.4. For a compact Hausdorff space $X$, let $C(X)$ denote the Banach algebra with supnorm $\|f\|_{\infty}=\sup \{|f(x)| \mid x \in X\}$ of all continuous complexvalued functions on $X$. Let $|\cdot|$ be any norm on $C(X)$ such that $(C(X),|\cdot|)$ is a normed linear space (not necessarily complete) satisfying $\left|f^{2}\right|=|f|^{2}$ for all $f$ in $C(X)$. Then $|\cdot|=\|\cdot\|_{\infty}$.

Proof. By [3], $|\cdot|$ satisfies $|f g| \leq|f||g|$ for all $f, g$; hence, $(C(X),|\cdot|)$ is a normed algebra with the result $\|\cdot\|_{\infty} \leq|\cdot|[11$, Theorem 1.2.4]. By Corollary $2.3,|\cdot| \leq r(\cdot)=\|\cdot\|_{\infty}$.

Corollary 2.5. Let $A$ be an sb algebra, B be a complete barreled uT-algebra, and $\phi: A \rightarrow B$ be a surjective homomorphism. Then the topology of $B$ is normable. Proof. By Theorem 2.1, $B=\phi(A)=b(B)$, and the conclusion follows by applying the open mapping theorem to the identity map $i:\left(b(B),\|\cdot\|_{\infty}\right) \rightarrow B$.

\section{REMARKS}

(3.1) In Theorem 2.1 the assumption that $A$ is a $Q$-algebra cannot be omitted, even if $A$ is a complete uT-algebra and $B$ is a uB-algebra. Consider the complete $\mathrm{uT}$-algebra $(C[0,1], \tau)$ with the topology $\tau$ of uniform convergence on all countable compact subsets of $[0,1]$. It is not a $Q$-algebra, because the topology of a complete uT-algebra, which is a $Q$-algebra, has to 
be normable [3, Theorem 2], and $\tau$ fails to be normable. The identity map $i:(C[0,1], \tau) \rightarrow\left(C[0,1],\|\cdot\|_{\infty}\right)$ is not continuous.

(3.2) In Corollary 2.5 the assumption that $B$ be barreled cannot be omitted. This is seen by considering the identity map $i:\left(C[0,1],\|\cdot\|_{\infty}\right) \rightarrow(C[0,1], \tau)$, where $\tau$ is as in (3.1).

(3.3) In Theorem 2.1 the hypothesis that $B$ is a uT-algebra cannot be omitted, even if $A$ is a $Q$-normed algebra and $B$ is a complete metrizable lmc $Q$ algebra. Take $A=\left(C^{\infty}[0,1],\|\cdot\|_{\infty}\right)$, the algebra of all $C^{\infty}$-functions on $[0,1]$ with the supnorm $\|\cdot\|_{\infty}$. For any $f \in A, \operatorname{sp}_{A}(f)=$ range of $f$; hence, $r_{A}(f)=\|f\|_{\infty}=\lim \sup _{n \rightarrow \infty}\left\|x^{2^{n}}\right\|_{\infty}^{1 / 2^{n}}=\lim \sup _{n \rightarrow \infty}\left\|f^{n}\right\|_{\infty}^{1 / n}$, so by $[2$, Proposition 15], the normed algebra $A$ is a $Q$-algebra. Let $B=\left(C^{\infty}[0,1], t\right)$, a complete lmc algebra with topology $t$ defined by submultiplicative norms

$$
p_{n}(f)=\sup _{0 \leq t \leq 1}\left[\sum_{0}^{n} \frac{\left|f^{(k)}(t)\right|}{k !}\right]
$$

it is a $Q$-algebra [9, Appendix E]. The identity map $i: A \rightarrow B$ is not continuous. (3.4) The topological algebra $B$ in (3.3) is not a uT-algebra, for otherwise, $B$ being a $Q$-algebra, the topology $t$ has to be normable [3, Theorem 2], with the result that $B$ has to be a Banach algebra. On the other hand, the algebra $B$ fails to be a Banach algebra under any norm, as a semisimple commutative Banach algebra is known not to admit a nonzero derivation. Thus, the discontinuity of the identity map $i: A \rightarrow B$ in (3.3) also shows that in Corollary 2.5 the assumption that $B$ is a $\mathrm{uT}$-algebra cannot be omitted.

(3.5) In Corollary 2.4 the square property $\left|f^{2}\right|=|f|^{2} \quad(f \in C(X))$ of the norm $|\cdot|$ cannot be omitted (or weakened to square inequality). By [5], for an infinite compact Hausdorff space $X$, there exists a norm on $C(X)$, distinct from $\|\cdot\|_{\infty}$ and not equivalent to it, making $C(X)$ an incomplete normed algebra. Also,

$$
|f|=\sup \left\{\frac{|f(s)+f(t)|}{2}+\frac{|f(s)-f(t)|}{2} \mid s, t \text { in } X\right\}
$$

defines a norm on $C(X)$, equivalent to $\|\cdot\|_{\infty}$ but distinct from $\|\cdot\|_{\infty}$, making $C(X)$ a Banach algebra satisfying $\|\cdot\|_{\infty} \leq|\cdot| \leq 2\|\cdot\|_{\infty}[4$, Example 7.5, p. 70] and hence satisfying the square inequality $\frac{1}{4}|f|^{2} \leq\left|f^{2}\right| \leq|f|^{2}$ for all $f \in C(X)$.

(3.6) Corollary 2.4 does not hold for uniformly closed nonselfadjoint subalgebras of $C(X)$. On the supnorm disc algebra $A(D)$ of all those continuous functions on the closed unit disc $D$ in the complex plane that are analytic in the interior of $D,|f|_{r}=\sup \{|f(z)||0<| z \mid \leq r\}, 0<r<1$, define uniform norms distinct from the supnorm $\|\cdot\|_{\infty}$, satisfying $|\cdot| r \leq\|\cdot\|_{\infty}$. However, it follows [3] that if $|\cdot|$ is a uniform norm on a uB-algebra $(A,\|\cdot\|)$ such that either $(A,\|\cdot\|)$ is regular or $(A,|\cdot|)$ is a $Q$-algebra, then $|\cdot|=\|\cdot\|_{\infty}$.

(3.7) In Theorem 2.2 the hypothesis that $A$ is regular cannot be omitted, even if $A$ and $B$ are uB-algebras. Take $A$ to be the supnorm disc algebra $A(D)$ as in (3.6). Let $0<r<1, \Gamma=\{z \in D|| z \mid=r\}, B=\left(C(\Gamma),\|\cdot\|_{\infty}\right)$, and $\phi: A \rightarrow B$ be $\phi(f)=\left.f\right|_{\Gamma}$. The algebra $A$ is not regular $[8, \S 7.2$, p. 167], and $\phi^{-1}$ fails to be continuous. 
(3.8) Let us note that Theorems 2.1 and 2.2 are $\mathrm{uT}$-algebra analogues of a couple of automatic continuity results for *-homomorphisms between LMC* $^{*}$ algebras proved in [6].

\section{REFERENCES}

1. G. R. Allan, A spectral theory for locally convex algebras, Proc. London Math. Soc. 15 (1965), 399-421.

2. S. J. Bhatt, On spectra and numerical ranges in locally m-convex algebras, Indian J. Pure Appl. Math. 14 (1983), 596-603.

3. S. J. Bhatt and D. J. Karia, Uniqueness of the uniform norm with an application to topological algebras, Proc. Amer. Math Soc. 116 (1992), 499-504.

4. F. F. Bonsall and J. Duncan, Numerical ranges of operators on normed spaces and of elements of normed algebras, London Math. Soc. Lecture Notes Ser., vol. 2, Cambridge Univ. Press, Cambridge, New York, and Melbourne, 1971.

5. H. G. Dales, $A$ discontinuous homomorphism from $C(X)$, Amer. J. Math. 101 (1979), 647-734.

6. Maria Fragoulopoulou, Automatic continuity of *morphisms between nor-normed topological *algebras, Pacific J. Math. 14 (1991), 57-70.

7. A. Guichardet, Special topics in topological algebras, Gordon and Breach, New York, London, and Paris, 1968.

8. R. Larsen, Banach algebras, Marcel Dekker, New York, 1973.

9. E. A. Michael, Locally multiplicatively convex topological algebras, Mem. Amer. Math. Soc., No. 11, Amer. Math. Soc., Providence, RI, 1952.

10. W. Rudin, Functional analysis, Tata-McGraw Hill, New Delhi, 1973.

11. S. Sakai, $C^{*}$ - and $W^{*}$-algebras, Ergeb. Math. Grenzgeb., vol 60, Springer-Verlag, Berlin, Heidelberg, and New York, 1971.

Department of Mathematics, Sardar Patel University, Vallabh Vidyanagar, 388 120, GUJARAT, INDIA 